\title{
Study on Hydraulic Performances of a 3-Bladed Inducer Based on Different Numerical and Experimental Methods
}

\author{
Yanxia Fu, ${ }^{1}$ Yujiang Fang, ${ }^{2}$ Jiangping Yuan, ${ }^{2}$ Shouqi Yuan, ${ }^{2}$ \\ Giovanni Pace, ${ }^{3}$ and Luca Dagostino ${ }^{4}$ \\ ${ }^{1}$ School of Energy and Power Engineering, Jiangsu University, No. 301, Xuefu Road, Zhenjiang, Jiangsu 212013, China \\ ${ }^{2}$ National Research Centre of Pumps, Jiangsu University, No. 301, Xuefu Road, Zhenjiang, Jiangsu 212013, China \\ ${ }^{3}$ Alta S.p.A., 5 Via Della Gherardesca, Ospedaletto, 56121 Pisa, Italy \\ ${ }^{4}$ Civil and Industrial Engineering Department, University of Pisa, 2 Largo L. Lazzarino, 56121 Pisa, Italy
}

Correspondence should be addressed to Yanxia Fu; yanxiafu@ujs.edu.cn

Received 6 June 2016; Revised 16 September 2016; Accepted 28 September 2016

Academic Editor: Jechin Han

Copyright (C) 2016 Yanxia Fu et al. This is an open access article distributed under the Creative Commons Attribution License, which permits unrestricted use, distribution, and reproduction in any medium, provided the original work is properly cited.

\begin{abstract}
The hydraulic performances of a 3-bladed inducer, designed at Alta, Pisa, Italy, are investigated both experimentally and numerically. The 3D numerical model developed in ANSYS CFX to simulate the flow through the inducer and different lengths of its inlet/outlet ducts is illustrated. The influence of the inlet/outlet boundary conditions, of the turbulence models, and of the location of inlet/outlet different pressure taps on the evaluation of the hydraulic performance of the inducer is analyzed. As expected, the predicted hydraulic performance of the inducer is significantly affected by the lengths of the inlet/outlet duct portions included in the computations, as well as by the turbulent flow model and the locations of the inlet/outlet pressure taps. It is slightly affected by the computational boundary conditions and better agreement with the test data obtained when adopting the $k$ - $\omega$ turbulence model. From the point of the pressure tap locations, the pressure rise coefficient is much higher when the inlet/outlet static pressure taps were chosen in the same locations used in the experiments.
\end{abstract}

\section{Introduction}

Propellant feed turbopumps are essential components of all primary propulsion concepts powered by liquid propellant rocket engines. Due to the severe limitations imposed to Space Transportation Systems, liquid propellant feed turbopumps must meet extremely demanding pumping, suction, and reliability requirements $[1,2]$. In these machines inducers are routinely employed to prepressurize the inlet flow to the main centrifugal stage(s), thereby improving the suction performance and reducing the propellant tank pressure and weight [3]. Usually inducers used in space applications show fewer blades than centrifugal pumps. Other characteristics of inducers include low values of the working flow coefficient, large stagger angles, and high blade solidity. The typical small angles of incidence, the long blades, and the usually increasing pitch between blades cause a reduction of blade loading. These features allow for significant improvements of the performance in cavitating conditions. Nevertheless, this geometry shows some drawbacks, such as highly viscous and turbulent flow inside the blade channels. It must be emphasized that the effect of viscosity on pumping degradation is of secondary importance in space inducers, given that most of the pressure rise is due to the centrifugal stage and the role of the inducer is mostly sacrificial so that cavitation effects can be reduced as much as possible on the main impeller.

Ongoing improvements of computational fluid dynamics (CFD) are rapidly promoting its role and use as an effective tool for research and development of hydraulic machinery with respect to complex and expensive experimentation [4]. Specifically, the choice of the boundary conditions, of the mesh size, and of the turbulence model is known to be the main aspects critically affecting the computational efficiency in the simulation of both noncavitating and cavitating flows.

Computations have been carried out by means of the commercial ANSYS CFX 14.5 software package installed at 
TABle 1: The parameters of Blade High-Performance Computing Clusters System.

\begin{tabular}{|c|c|}
\hline CPU & $\begin{array}{l}\text { 2x Intel Xeon X5650 CPU with } 6 \text { physical cores } \\
\text { for each, } 2.40 \mathrm{GHz}, 12 \mathrm{M} \text { Three-level buffer }\end{array}$ \\
\hline Memory & $\begin{array}{l}24 \text { G DDR3 RDIMM, } 18 \text { slots, the maximum } \\
\text { memory } 384 \text { G }\end{array}$ \\
\hline Hard risk & 2x 146 G 10K SAS 6 G 2.5, Hot-Swap hard disk \\
\hline Blade server & Dawning Blade Full View Manager System \\
\hline $\begin{array}{l}\text { Operating } \\
\text { system }\end{array}$ & SUSE Linux Enterprise Server 11 for 64-bit \\
\hline
\end{tabular}

Jiangsu University, Zhenjiang City, China, in the Blade HighPerformance Computing Clusters System, whose main operational parameters are listed in Table 1. Reference validation experiments have been carried out in the CPRTF (Cavitating Pump Rotordynamic Test Facility) at ALTA, Pisa, Italy [5], on the three-bladed DAPROT3 inducer, designed as described in d'Agostino et al. [6,7] and manufactured in 7075-T6 aluminum alloy [8]. DAPROT3 inducer is designed to be a high-head axial inducer with tapered-hub and variable pitch, and the main design characteristics are summarized in Table 2.

\section{Numerical Methods}

2.1. Geometrical Data and Mesh Generation. The computational model includes the three-bladed inducer, whose main dimensions and characteristics are reported in Table 2, together with suitably long portions (20 diameters upstream and downstream) of the inlet and outlet ducts, as shown in Figure 1. Unstructured tetrahedral cells have been used for the numerical mesh in consideration of their more rapid generation with ANSYS ICEM CFD 14.5 and wider adaptability to the complex geometry of the test inducer with twisted blades and internal flow passages. Structure meshes were generated for the inlet and outlet pipes. The number of grid nodes was systematically increased throughout the whole computational domains such that six grids were generated as shown in Table 3. Finally, in mesh number 6, used to investigate the sensitivity of the simulations to the inclusion of shorter inlet/outlet duct lengths, only unstructured tetrahedral elements have been employed for all of the inducer's computational domains.

2.2. Governing Equations and Turbulence Models. ANSYS CFX uses a finite volume formulation to solve the RANS momentum, continuity and turbulence equations for incompressible, turbulent flow in the inducer. Since it was originally proposed by Launder and Spalding [9], the standard $k-\varepsilon$ turbulence model has become the workhorse of engineering flow calculations. The $k-\varepsilon$ turbulence model of ANSYS CFX uses the scalable wall-function approach to improve robustness and accuracy of the computations near the walls, where the mesh is very fine. This feature allows for the solution to be obtained on arbitrarily fine grids and represents a significant improvement over the standard wall function approach. In the present study, four turbulence models (namely, the $k-\omega$, RNG $k-\varepsilon, k-\varepsilon$ ERAES, $k-\varepsilon$ models) have been chosen to investigate their influence on the prediction of the hydraulic performances of the inducer when operating at $90 \%$ of its design flow rate $\left(Q_{D}\right)$.

2.3. Boundary Conditions. Since the computational cost is known to depend also on the inlet/outlet boundary conditions, it is important to investigate the impact [10]. Here two sets of boundary conditions have been taken into consideration by imposing in

set $Q-P$ : the upstream mass flow rate and downstream static pressure;

set $P$-Q: the upstream total pressure and downstream mass flow rate;

respectively.

\section{Experimental Apparatus}

The Cavitating Pump Rotordynamic Test Facility (CPRTF, ALTA S.p.A., Pisa, Italy; Figure 2) is a versatile and easily instrumentable facility operating in water at temperatures up to $90^{\circ} \mathrm{C}$ [11]. The inlet pressure is monitored by two absolute transducers positioned: one is placed about one and half diameter upstream of the blade leading edge and the other one is placed in a moved back position, about six diameters upstream of the blade leading edge, in order to consider possible prerotation effects on inlet pressure. Both the absolute transducers are Druck transducers (model PMP $1400,0-1.5$ bar operating range, $0.25 \%$ precision class). A pair of redundant differential pressure transducers measure the pump pressure rise between the same inlet section and an outlet section positioned about two diameters downstream of the blade trailing edges (Kulite, model BMD 1P 1500 100, 06.8 bar-d operating range, $0.1 \%$ precision class; Druck, model PMP 4170, 0-1 bar-d operating range, $0.08 \%$ precision class).

3.1. Test Item. Type The DAPROT3 inducer used in the experimental tests has been designed by means of the well validated reduced-order model developed by Alta for the inducer design and performance prediction of axial inducers [12]. It is a three-bladed high-head inducer with taperedhub and variable pitch, made of 7075-T6 aluminum alloy with Sanford surface treatment. The geometry generated by the model is consistent with the typical geometries and operational characteristics of modern space rocket inducers. The moderate blade loading and relatively high solidity have been chosen for reducing the leading edge cavity and improving the suction performance. An incidence-to-blade angle ratio $\alpha / \beta_{b}<0.5$ has been selected with the aim of controlling the occurrence of surge instabilities at design flow under cavitating conditions. Table 2 shows some pictures of the DAPROT3 inducer. A series of tests have been carried out on this three-bladed axial inducer at different flow coefficients under noncavitating conditions. 
TABLE 2: Geometrical and operational parameters of the DAPROT3 inducer.

\begin{tabular}{lccc}
\hline Design flow coefficient & {$[-]$} & $\Phi_{D}$ & 0.065 \\
Number of blades & {$[-]$} & $N$ & 3 \\
Tip radius & $\mathrm{mm}$ & $r_{T}$ & 81.0 \\
Inlet tip blade angle & $\mathrm{deg}$ & $\gamma_{\mathrm{Tle}}$ & 82.10 \\
Inlet hub radius (fully developed & $\mathrm{mm}$ & $r_{\mathrm{Hle}}$ & 44.5 \\
blade) & $\mathrm{mm}$ & $r_{\mathrm{Hte}}$ & 65.6 \\
Outlet hub radius & $\mathrm{mm}$ & $c_{a}$ & 63.5 \\
Axial length (fully developed blade) & $\mathrm{rpm}$ & $r_{\mathrm{H}}$ & 35.0 \\
Rotational speed & $\mathrm{mm}$ & 90.0 \\
Inlet hub radius & $\mathrm{mm}$ & 25.95 \\
Axial length & $\mathrm{mm}$ & $h_{m}$ & 0.47 \\
Mean blade height & {$[-]$} & $D$ & 0.33 \\
Diffusion factor & {$[-]$} & $\alpha / \beta_{b}$ & \\
Ratio between the incidence and blade & {$[-]$} & $\sigma_{T}$ & 1.68 \\
angles $\left(\beta_{b}=\pi / 2-\gamma_{\text {Tle }}\right)$ & $\alpha$ & 2.58 \\
Tip solidity & $\mathrm{deg}$ & $\gamma_{\mathrm{Tte}}$ & 70.56 \\
Incidence tip angle @ design & $\mathrm{deg}$ & & \\
Outlet tip blade angle & &
\end{tabular}

TABLE 3: Details of mesh elements.

\begin{tabular}{|c|c|c|c|c|c|c|}
\hline \multirow{2}{*}{ Computational domains } & \multicolumn{6}{|c|}{ Mesh elements } \\
\hline & Case 1 & Case 2 & Case 3 & Case 4 & Case 5 & Case 6 \\
\hline Inlet & & & 624134 & & & 7234222 \\
\hline Outlet & & & 296934 & & & 469918 \\
\hline Inducer & 820816 & 1340548 & 1548792 & 1762226 & 1762586 & 3675103 \\
\hline All domains & 1741884 & 2261616 & 2469860 & 2683294 & 2683654 & 11379243 \\
\hline
\end{tabular}

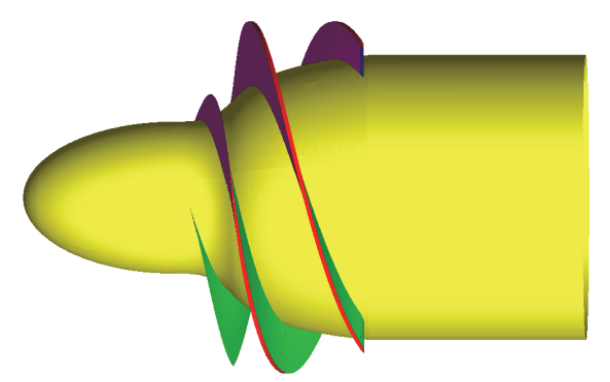

(a)

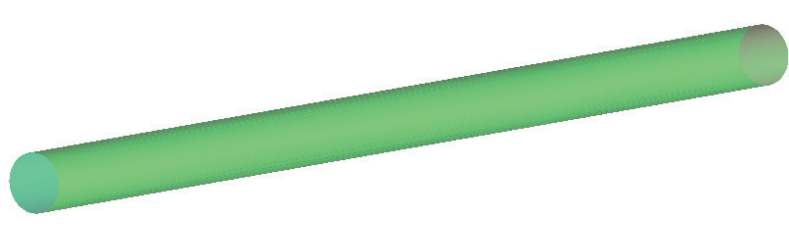

(b)

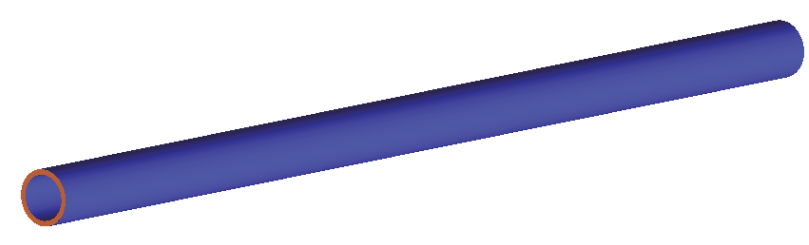

(c)

Figure 1: (a) A 3D view of the inducer; (b) the extended inlet duct; (c) the outlet duct. 

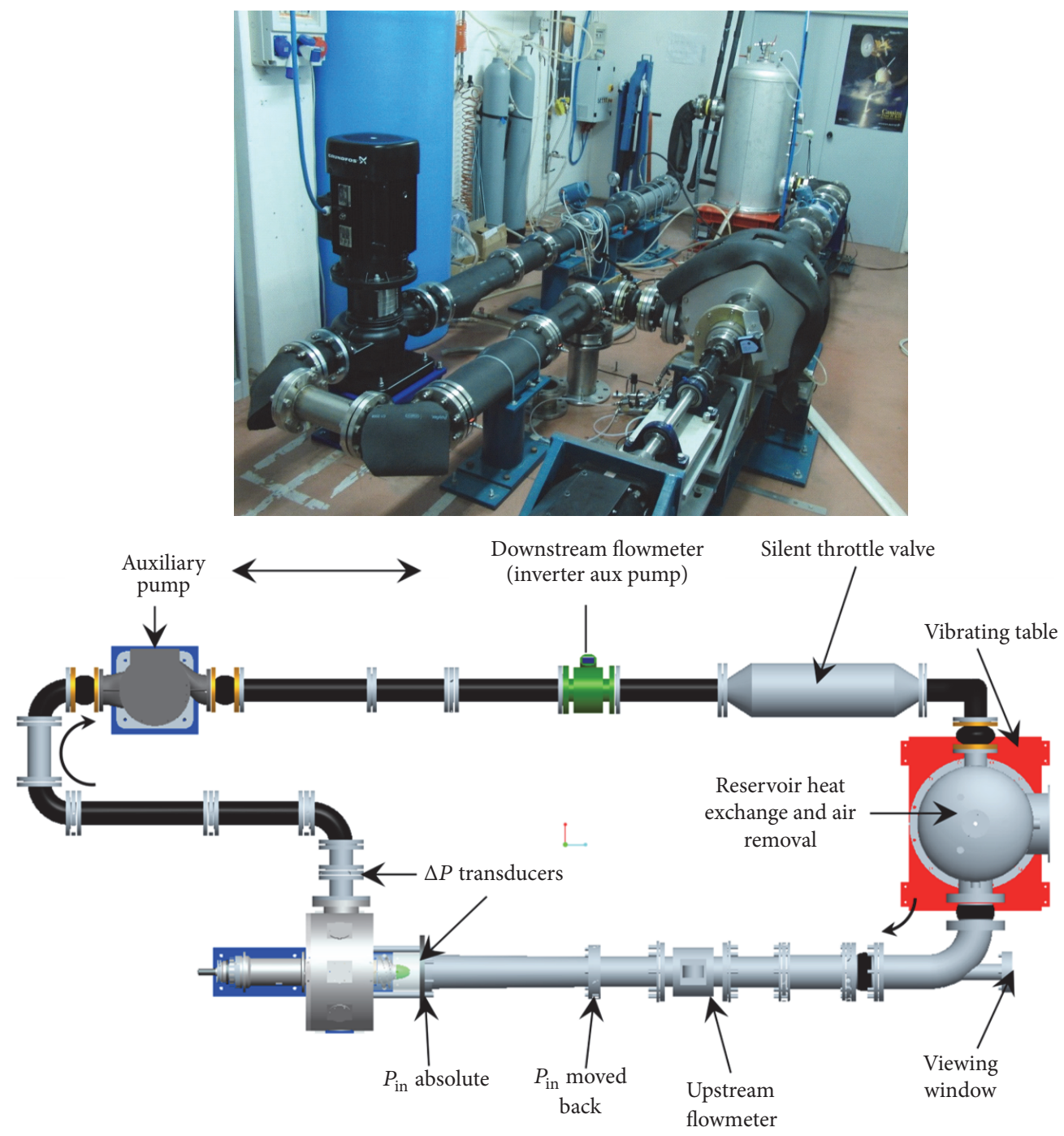

Figure 2: The Cavitating Pump Rotordynamic Test Facility and the experimental setup.

3.2. Experimental Results. The characterization of the pumping performance of the DAPROT3 has been assessed throughout a series of tests conducted in water at room temperature $\left(20^{\circ} \mathrm{C}\right)$ and at inlet static pressure well above the cavitation inception conditions. The pumping performance has been evaluated in terms of static head coefficient $\Psi=$ $\Delta P / \rho \Omega^{2} r_{T}^{2}$, with the pressure rise measured at the locations shown in Figure 3, as a function of the flow coefficient $\Phi=Q / \pi \Omega r_{T}^{3}$. All experiments have been carried out at Reynolds numbers $\left(\operatorname{Re}=2 \Omega r_{T}^{2} / \nu\right)$ higher than $10^{6}$ for the results to be virtually independent of turbulent effects, as confirmed by Brennen [13] in a series of tests at different rotating speeds. Figure 3 shows the configuration used for the experimental characterization of the DAPROT3 inducer. They have been reported for later comparison with the numerical simulations.

\section{Results and Discussions}

4.1. Convergence Criteria. Simulations have been typically considered to have reached convergence when the residuals of the mass continuity equation fell below $1 \times e^{-5}$. In addition, convergence has also been checked by monitoring a number of relevant integrated quantities, such as by verifying the constancy of the average flow pressure at the reference outlet cross-section of the computational domain. An example of the typical results obtained in this way is illustrated in Figure 4, which shows that satisfactory convergence has been attained in the case under consideration both in terms of the residuals of the conservation equations and in terms of the regularity of the mean value of the outlet flow pressure.

4.2. Grid Refinement and Validation. Five different computational meshes have been taken into consideration and their resolutions have been optimized based on a sensitivity analysis on the hydraulic performance of the DAPROT3 inducer. Finally, these meshes have been used to assess gridindependence of the simulations.

The effects of the number of grid elements and of the boundary conditions on the prediction of the inducer performance operating at a flow coefficient of $\Phi=0.05903$ has been assessed. The results illustrated in Figure 5 indicate 


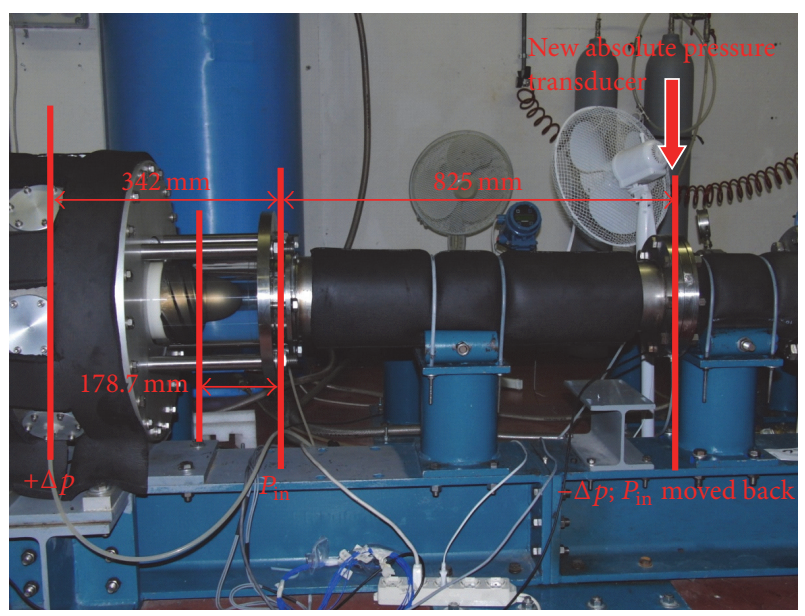

Figure 3: Test section setup for the characterization of the noncavitating performance of the inducer.

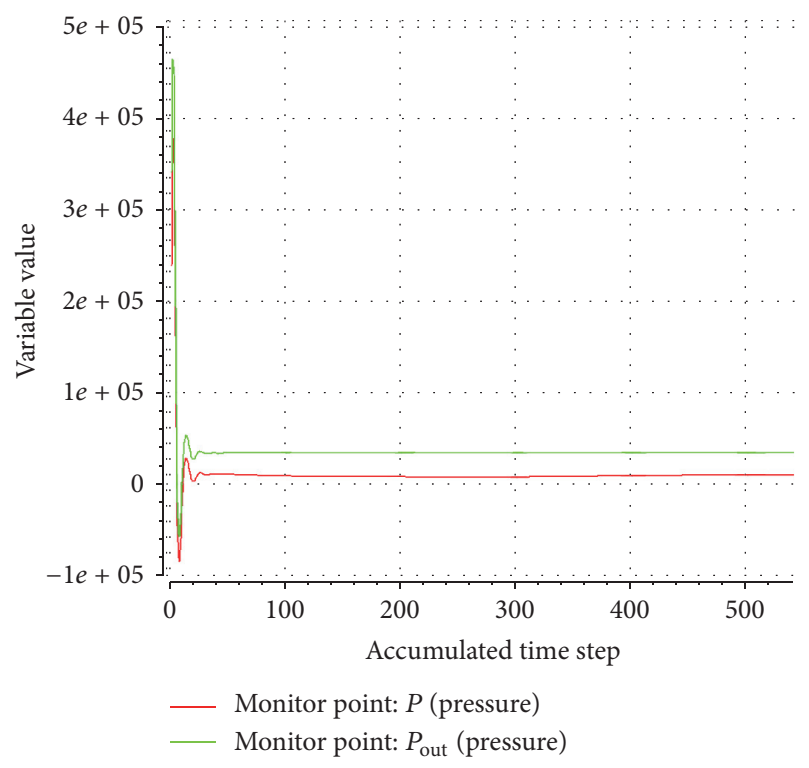

FIgURE 4: Convergence curves for monitors (mesh case 1 to 5).

that grids with intermediate and fine resolutions (from mesh 2 to 5) give very similar values for the static pressure rise coefficient of the inducer, while those obtained with coarse mesh (mesh 1) are significantly different and also depend on how the boundary conditions have been imposed (red and black histograms). Figure 5 also shows that the static pressure rise coefficient increases slowly with the number of grid elements and its dependence on the boundary conditions decreases. Both of these effects become virtually insignificant for mesh numbers larger than 176000 .

\subsection{Hydraulic Performances of the Inducer}

4.3.1. Comparison of Positions of Pressure Monitors. Simulations of the fully wetted flow through the DAPROT3 inducer operating at $90 \%$ of its design flow $(\Phi=0.05903)$ with

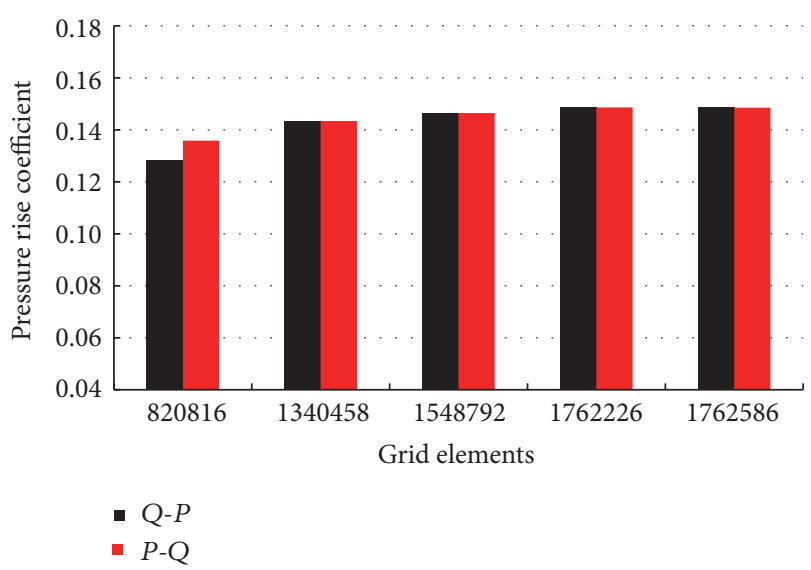

FIGURE 5: Grid independence check (mesh case 1 to 5).

$0.8 \mathrm{~mm}$ blade tip clearance have been carried out using the above five meshes and two sets of boundary conditions. Hereafter the results are discussed and validated by comparison with the pertinent experiments carried out in the CPRTF at Alta. Since the complex flow occurs in the inlet pipe [1417], the influence of the location of the reference stations for the evaluation of the inducer hydraulic performance has been investigated by considering both local and mean values of the static pressure on the relevant inlet/outlet flow cross-sections (indicated above as positions 1,2 , and 3). In particular, with reference to Figure 6(a), four positions of the nominal inlet/outlet stations in the computational domain have been used:

(1) Position 1: one diameter upstream of the blade leading edge for the inlet station and one diameter downstream of the blade trailing edge for the outlet station, corresponding to the locations where the inlet and outlet pressure transducers have been installed in the experimental tests on the inducer with $0.8 \mathrm{~mm}$ blade tip clearance.

(2) Position 2: 20 duct diameters upstream/downstream of the blade leading/trailing edges.

(3) Position 3: two diameters upstream of the blade leading edge for the inlet station and 2.5 diameters downstream of the blade trailing edge for the outlet station, corresponding to the locations where the inlet/outlet pressure transducers have been installed in the experimental tests on the inducer with $2 \mathrm{~mm}$ blade tip clearance.

(4) Position 4: six diameters upstream of the blade leading edge for the inlet station and one diameter downstream of the blade trailing edge, corresponding to the locations where the inlet/outlet pressure transducers have been installed in the experimental tests in order to eliminate prerotation effects.

As expected, the results shown in Figure 6(b) confirm that the static pressure rise coefficients $\Psi$ predicted by fine-mesh simulations (mesh 4 in this case) for positions from 1 to 3 of the nominal inlet/outlet stations of the inducer are lower 


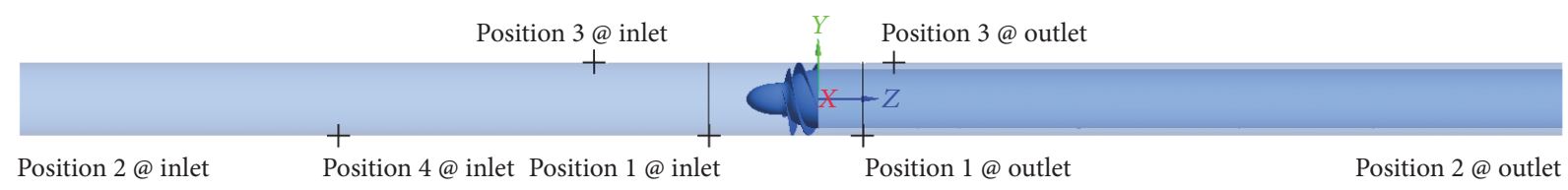

(a) Distributions of pressure monitors

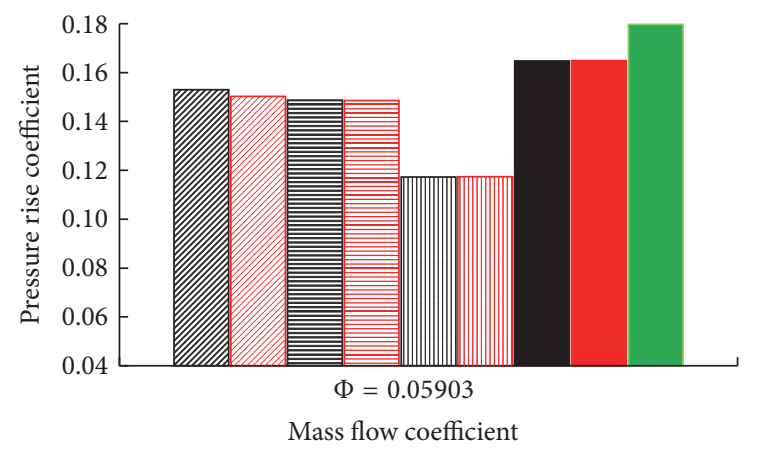

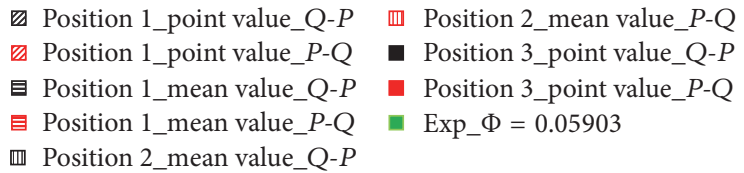

(b) Comparison of four different pressure monitors
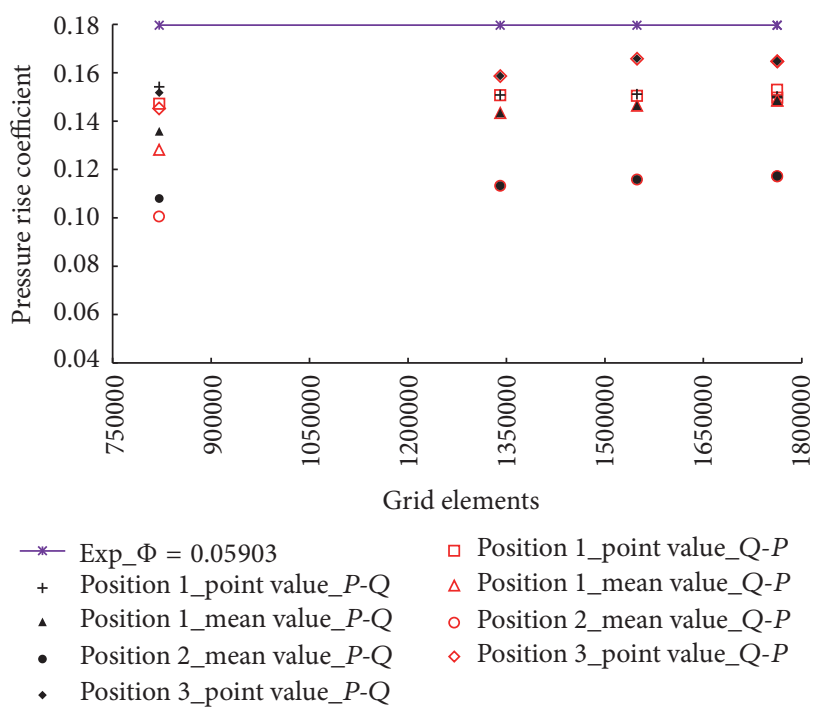

(c) Comparison of grid elements and four different pressure monitors

FIGURE 6: Comparison of different pressuring measuring positions at the flow coefficient of $\Phi=0.05903$.

than the experimental value. The static head coefficients computed from local values of the pressure are slightly higher than those evaluated using mean pressure values. Figure 6(c) compares the static pressure rise coefficient obtained from simulations on meshes from 1 to 5 for sets $Q-P$ and $P-Q$ of the boundary conditions and positions 1, 2 and, 3 of the reference inlet/outlet stations of the inducer. The values of the pressure rise computed on each mesh for different inlet/outlet positions and sets of boundary conditions display a small dispersion, corresponding to an error of about $0.02 \%$. In all cases the predicted performance of the inducer is lower than the experimental value, which has been measured by locating the static pressure taps at position 1. Increasing deviations have been obtained when using positions 3,1 , and 2 of the nominal inlet/outlet stations for the evaluation of the inducer pressure rise. In particular, in the simulations on finer meshes the relative deviations for these positions have been computed to be equal to $-8.28 \%,-14.87 \%$, and $-34.76 \%$, respectively. The same trend is also manifested in the simulations on coarser grids.

4.3.2. Comparison of Turbulence Models. Four turbulence models (namely, $k-\omega$, RNG $k-\varepsilon, k-\varepsilon$ ERAES, $k-\varepsilon$ ) have been used, in combination with the above two sets of boundary conditions, to investigate their influence on the observed discrepancies between numerical and experimental results obtained for the DAPROT3 inducer operating with $0.8 \mathrm{~mm}$ blade tip clearance at $\Phi=0.05903$. In accordance with the indications of the grid independence analysis, mesh 4 (with intermediate resolution) has been selected for the simulations in order to reduce their computational complexity and cost. Position 3 has been used to evaluate the inducer performance, since previous results indicated it provided better agreement with the experimental data. As indicated in Figure 7(b), the relative deviations from the experimental data of the simulated values of the pressure rise coefficient are $-3.99 \%$, $-7.79 \%,-4.38 \%$, and $-8.28 \%$ for the $k-\omega$, RNG $k-\varepsilon, k-\varepsilon$ ERAES, and $k-\varepsilon$ models, respectively. Hence, the pressure rise coefficient obtained using the $k-\omega$ turbulence model, combined with the inlet total pressure and outlet mass flow rate boundary conditions (set $P-Q$ ), better approximates the measured performance of the DAPROT3 inducer. The results obtained using the $k$ - $\omega$ model also appear to depend slightly on the choice of the boundary conditions, which, on the other hand, seems to have virtually no effect on the inducer performance obtained with the other three $k-\varepsilon$ turbulence models.

The static pressure pumping performance of the DAPROT3 inducer, operating at $0.8 \mathrm{~mm}$ blade tip clearance, has also been evaluated using the $k$ - $\omega$ turbulence model for comparison with the experiments. The results are displayed in Figure 7(c) for a number of positions of the reference inlet/outlet stations of the inducer. Either local values or cross-sectional averages of the static pressure have been used to evaluate the pressure rise coefficient. Since local pressure values are determined at the inner surface of the inducer casing, the use of cross-sectional averages reduces 


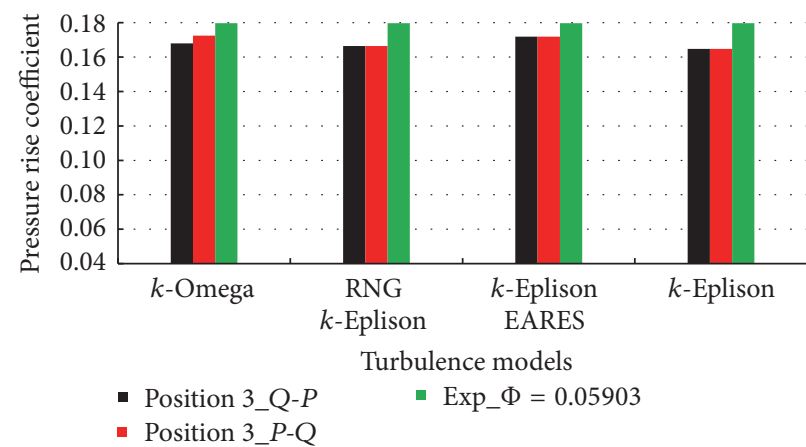

(a) Comparison of turbulence models and boundary conditions at the flow coefficient of $\Phi=0.05903$

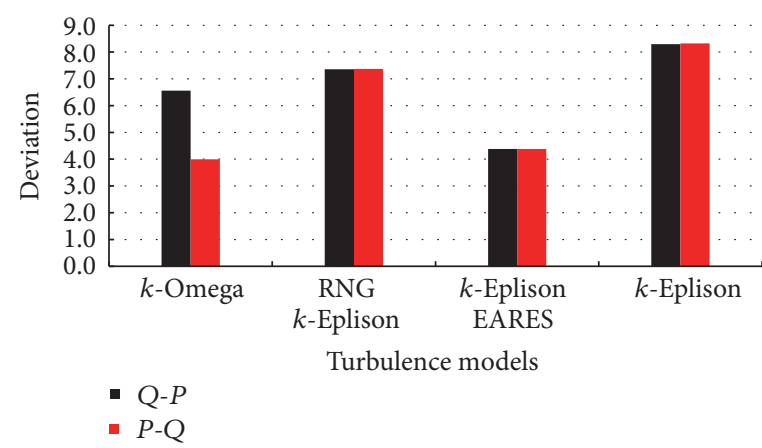

(b) Comparison of deviations of the pressure rise coefficient at the flow coefficient of $\Phi=0.05903$

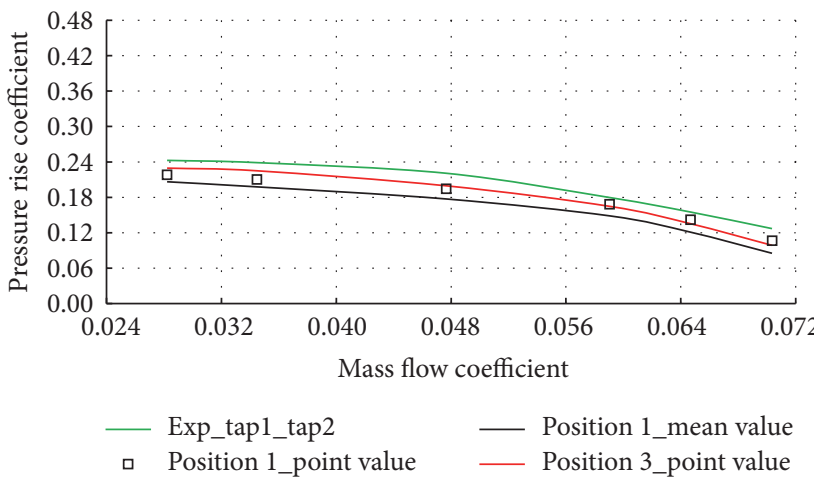

(c) Comparison of the hydraulic performances of the inducer

FIgURE 7: Comparison of different boundary conditions and different turbulence models.

the influence of centrifugal effects on the downstream pressure reading, decreasing the predicted value of the pumping performance. The two characteristics in Figure 7(c) evaluated at position 1 confirm this point. Finally, the same figure shows the inducer performance curves evaluated at positions 1 and 3, where the inlet pressure tap has been moved 5 duct diameters upstream. The two curves closely agree at higher flow rates, where the small difference of inlet side-wall friction is only responsible for their relative deviation. On the other hand, the sign of the deviation reverses at lower flow rates, corresponding to higher blade loading, and its magnitude increases as a consequence of the different intensity of the centrifugal effects induced on the inlet pressure readings by the occurrence of inducer flow prerotation.

\subsubsection{Comparison of Inlet and Outlet Ducts with Different} Lengths. According to the above analysis of the predicted inducer performance is still lower than the experimental one. Computations have therefore been extend to longer portions of the suction and discharge ducts with different lengths in order to better simulation the inlet/outlet flow and its influence on the inducer performance. In the shortest configuration the computational domain comprises inlet/outlet duct portions both 10 diameters and 15 diameters upstream and downstream of the inducer. In addition, a finer grid (mesh 6; see Table 3) has been generated for better consideration of the interactive effects with the other components of the computational model. The static pressure rise coefficient has been calculated using the local values of the flow pressure at the same locations where the inlet and outlet pressure transducers have been installed in the experimental tests on the DAPROT3 inducer with $0.8 \mathrm{~mm}$ blade tip clearance (position 1).

As expected, good agreement with the experimental performances is obtained in Figure 8(a) for the longest inlet/outlet duct length ( \pm 20 tip diameters), especially as the flow coefficient $\Phi$ is higher than $\Phi=0.04766$. By comparison, the simulations based on shorter inlet/outlet duct lengths on more refined grids (mesh 6) result in pressure rise coefficient systematically higher than experimental values and considerably longer computational times.

Next, the reference position for the evaluation of the inlet pressure has been located six diameters upstream of the leading edges of the inducer blades (position 4 in Figure 6) both in the computations and experiments, in order to eliminate the possible influence of backflow prerotation. The results, illustrated in Figure 8 (b) by solid lines, are compared with those previously obtained for position 1 (symbols). As expected, better agreement of the two pumping characteristics is attained especially at lower flow rates, where more intense backflow prerotation occurs as a consequence of higher blade loading. 


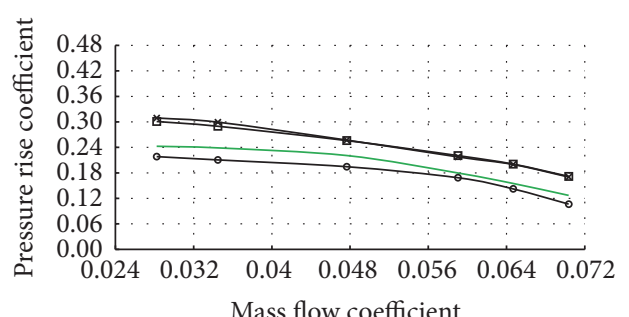

Mass flow coefficient

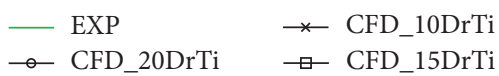

(a) Comparison of pump performances of the inducer based on three different lengths of inlet and outlet

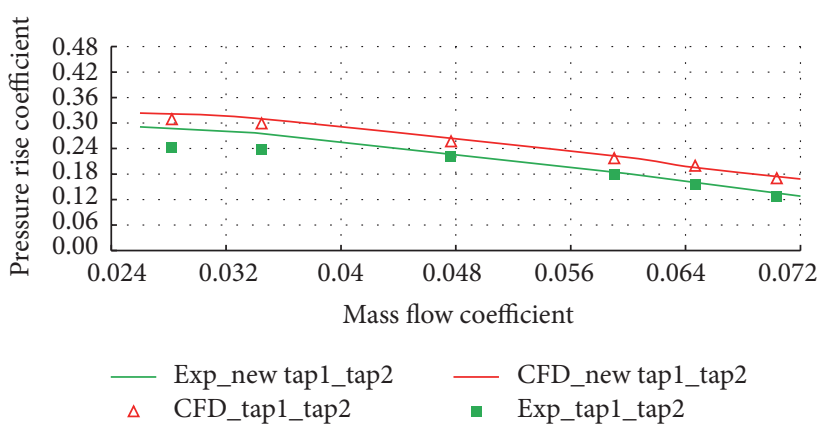

(b) Comparison of pump performances of the inducer based on the pressure taps located at position 4 based on experimental and CFD methods

Figure 8: Comparison of different lengths of the inlet and outlet pipes.

\section{Discussions and Conclusions}

The results of experimental and numerical pump performances of the inducer have been mainly analyzed. Moreover, the influences of four different turbulence models, 2 different boundary conditions as well as four different positions of inlet and outlet pressure taps were specially investigated in present simulations in comparison with the experiment. In addition, the different inner flow structures in the inducer in design and off-design flow rates are obtained.

From the present numerical analysis of the fully wetted flow in the DAPROT3 inducer and its comparison with the pertinent experimental results, the following conclusions have been drawn:

(1) The more refined mesh proved to be better capable of modeling the internal flow through the inducer. The influence of the boundary conditions on the prediction of the static pressure rise turned out to be small, while the lengths of inlet and outlet ducts did show to significantly affect the inducer performance prediction.

(2) Comparison with the experimental results indicated that the $k-\omega$ turbulence model proved to be the most accurate of the four alternatives examined in the present study. The two different sets of numerical boundary conditions turned out to have a small influence on the inducer performance predictions obtained from the use of $k-\varepsilon$ turbulence models.

(3) The large deviation between these $\Phi-\Psi$ curves was found at the flow rate coefficient lower than $\Phi=$ 0.04766 based on the longer portion of the inlet and outlet ducts with the length of 20 diameters of the blade tip radius. The reduced lengths of the inlet and outlet ducts significantly increase the hydraulic performances of the inducer and give confidence in predicting the hydraulic performances of the model inducer compared with the experimental data.

In summary, the simulated hydraulic performances of the test inducer agree well with the relevant experimental results over a wide range of operating conditions, indicating that the proposed numerical model and methods adequately capture the internal flow in the DAPROT3 inducer. Moreover, the different pressure tap positions used for measuring the inlet and outlet static pressure were first discussed both by numerical and experimental ways in this study. The model represents therefore an effective tool to understand, analyze, predict, and control the mechanisms of the complex phenomena taking place in the flow through inducers operating over a wide range of conditions above and below the design point.

\section{Nomenclature}

c: $\quad$ Tip blade clearance, $m$

D: Diffusion factor

L: Axial length, $\mathrm{m}$

$N$ : Number of blades

$\Omega$ : Inducer rotational speed, $\mathrm{rad} / \mathrm{s}$

$P$ : $\quad$ Static pressure, $\mathrm{Pa}$

Q: Volumetric flow rate, $\mathrm{m}^{3} / \mathrm{h}$

$\Phi$ : Flow coefficient, $\Phi=Q / \pi \Omega r_{T}^{3}$

$\Psi$ : Static head coefficient, $\Psi=\left(P_{2}-P_{1}\right) / \pi \Omega^{2} r_{T}^{2}$

$\delta$ : Tip clearance

$\gamma$ : Blade angle from axial direction

$\rho$ : Liquid density

$s$ : Azimuthal blade spacing

$\sigma: \quad$ Blade solidity $=c / s$

Re: Reynolds number, $\operatorname{Re}=2 \Omega r_{T}^{2} / \nu$

$r_{H}$ : Inducer hub radius, $\mathrm{m}$

$r_{T}$ : Inducer tip radius, $\mathrm{m}$

$h_{m}$ : Mean blade height, $\mathrm{m}$

$c_{a}$ : Mean blade height, $\mathrm{m}$

$\beta_{b}$ : Blade angle evaluated with respect to the normal to the axial direction.

\section{Subscripts}
$D$ : Design conditions
$T$ : Tip radius
le: Leading edge
te: Trailing edge 
v: Vapor pressure

1: Upstream station

2: Downstream station.

\section{Competing Interests}

The authors declare that they have no competing interests.

\section{Acknowledgments}

This study is supported by National Natural Science of China (nos. 51609107 and BK20160539), Jiangsu University Senior Personnel Scientific Research Foundation (no. 15JDG073). The present DAPROT3 experimental work has been carried out in Alta under ESA's support (no. 4000102585/10/NL/Sfe).

\section{References}

[1] L. Torre, A. Pasini, A. Cervone, and L. d'Agostino, "Experimental performance of a tapered axial inducer: comparison with analytical predictions," in Proceedings of the 45th AIAA/ASME/SAE/ASEE Joint Propulsion Conference \& Exhibit, Denver, Colo, USA, August 2009.

[2] L. Torre, A. Pasini, A. Cervone, and L. d'Agostino, "Experimental characterization of the rotordynamic forces on space rocket axial inducers," Journal of Fluids Engineering, vol. 133, no. 10, Article ID 101102, pp. 1-12, 2011.

[3] A. Cervone, G. Pace, L. Torre et al., "Effects of the leading edge shape on the performance of an axial three bladed inducer," in Proceedings of the 14th International Symposium on Transport Phenomena and Dynamics of Rotating Machinery (ISROMAC '12), Honolulu, Hawaii, USA, March 2012.

[4] J. H. Ferziger and M. Perić, Computational Methods for Fluid Dynamics, Springer, New York, NY, USA, 1996.

[5] G. Pace, L. Torre, A. Pasini, D. Valentini, and L. d'Agostino, "Experimental characterization of the dynamic transfer matrix of cavitating inducers," in Proceedings of the 49th AIAA/ASME/SAE/ASEE Joint Propulsion Conference, San Jose, Calif, USA, July 2013.

[6] A. Cervone, L. Torre, A. Pasini, and L. d'Agostino, "Cavitation and flow instabilities in a 4-bladed axial inducer designed by means of a reduced order analytical model," in Proceedings of the 47th AIAA/ASME/SAE/ASEE Joint Propulsion Conference, San Diego, Calif, USA, July-August 2011.

[7] D. Valentini, A. Pasini, G. Pace, L. Torre, and L. d'Agostino, "Experimental validation of a reduced order for radial turbopump design," in Proceedings of the 49th AIAA/ASME/SAE/ASEE Joint Propulsion Conference, San Jose, Calif, USA, July 2013.

[8] L. Torre, A. Pasini, A. Cervone, G. Pace, P. Miloro, and L. d'Agostino, "Effect of tip clearance on the performance of a three-bladed axial inducer," Journal of Propulsion and Power, vol. 27, no. 4, pp. 890-898, 2011.

[9] B. E. Launder and D. B. Spalding, "The numerical computation of turbulent flows," Computer Methods in Applied Mechanics and Engineering, vol. 3, no. 2, pp. 269-289, 1974.

[10] M. Karlsson, H. Nilsson, and J. O. Aidanpää, "Influence of inlet boundary conditions in the prediction of rotordynamic forces and moments for a hydraulic turbine using CFD," in Proceedings of the 12th International Symposium on Transport Phenomena and Dynamics of Rotating Machinery (ISROMAC '08), Honolulu, Hawaii, USA, February 2008.
[11] E. Rapposelli, A. Cervone, and L. d'Agostino, "A new cavitating pump rotordynamic test facility," in Proceedings of the 38th AIAA/ASME/SAE/ASEE Joint Propulsion Conference, Indianapolis, Ind, USA, July 2002.

[12] L. d'Agostino, L. Torre, A. Pasini, and A. Cervone, "On the preliminary design and noncavitating performance prediction of tapered axial inducers," Journal of Fluids Engineering, vol. 130, no. 11, Article ID 111303, pp. 1-8, 2008.

[13] C. E. Brennen, Hydrodynamics of Pumps, Concepts ETI Inc, Norwich, UK, Oxford University Press, Oxford, UK, 1994.

[14] L. Wang, C. Yang, B. Zhao, D. Lao, C. Ma, and D. Li, "The change of the inlet geometry of a centrifugal compressor stage and its influence on the compressor performance," Journal of Thermal Science, vol. 22, no. 3, pp. 197-208, 2013.

[15] Y. Z. Wu and H. Huang, "Experimental research of pressure distribution in the casing of inducer," Journal of Thermal Science, vol. 18, no. 4, pp. 301-305, 2009.

[16] K. Okita, H. Ugajin, and Y. Matsumoto, "Numerical analysis of the influence of the tip clearance flows on the unsteady cavitating flows in a three-dimensional inducer," Journal of Hydrodynamics, vol. 21, no. 1, pp. 34-40, 2009.

[17] C.-H. Choi, J.-S. Kim, and J. H. Kim, "Study on the forwardsweep inducer for LRE turbopumps," Acta Astronautica, vol. 65, no. 1-2, pp. 214-220, 2009. 


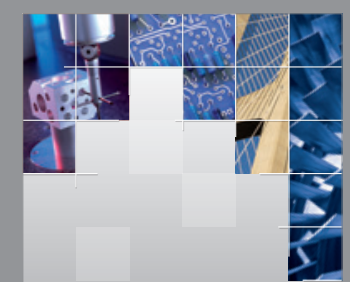

\section{Enfincering}
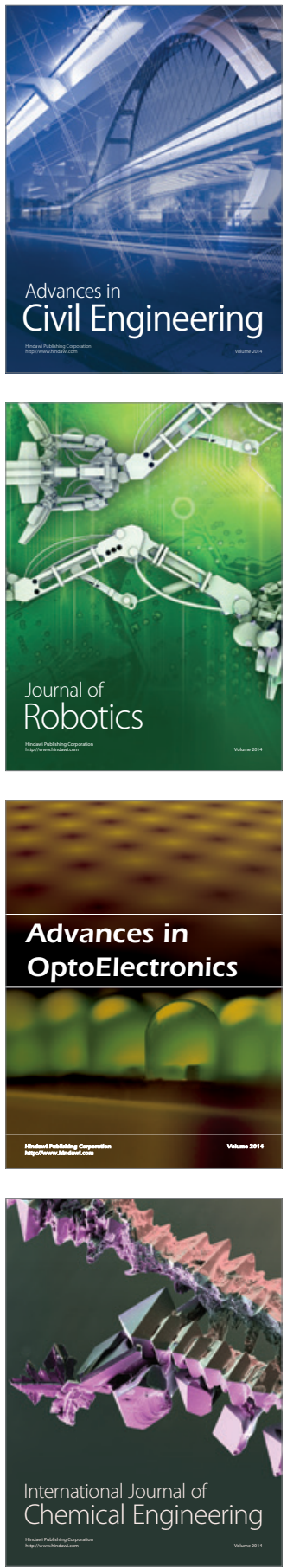

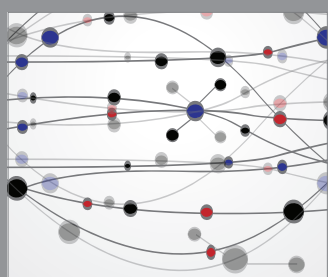

The Scientific World Journal

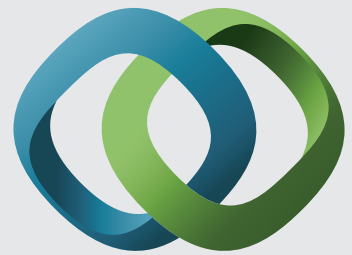

\section{Hindawi}

Submit your manuscripts at

http://www.hindawi.com
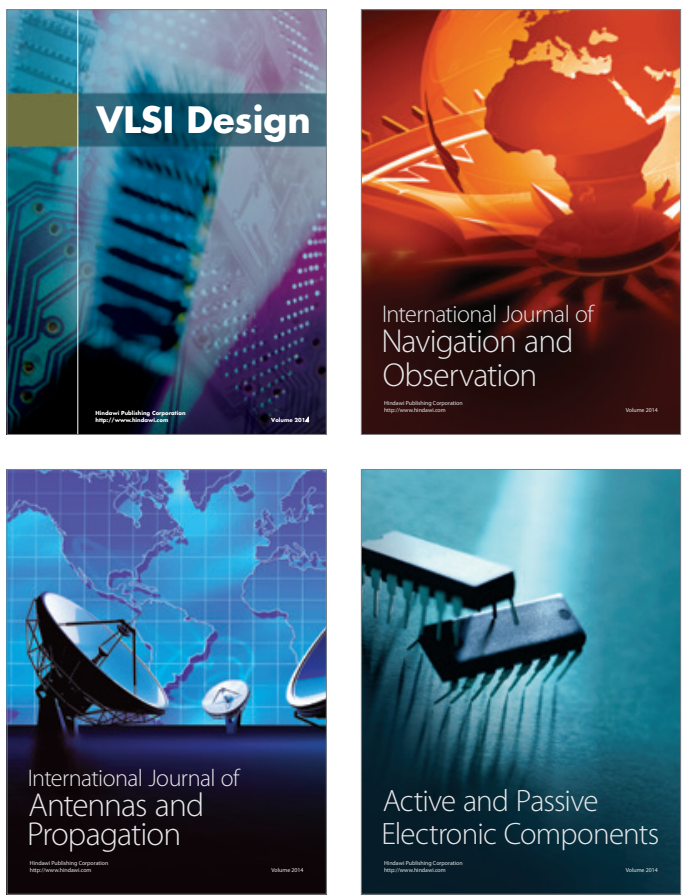
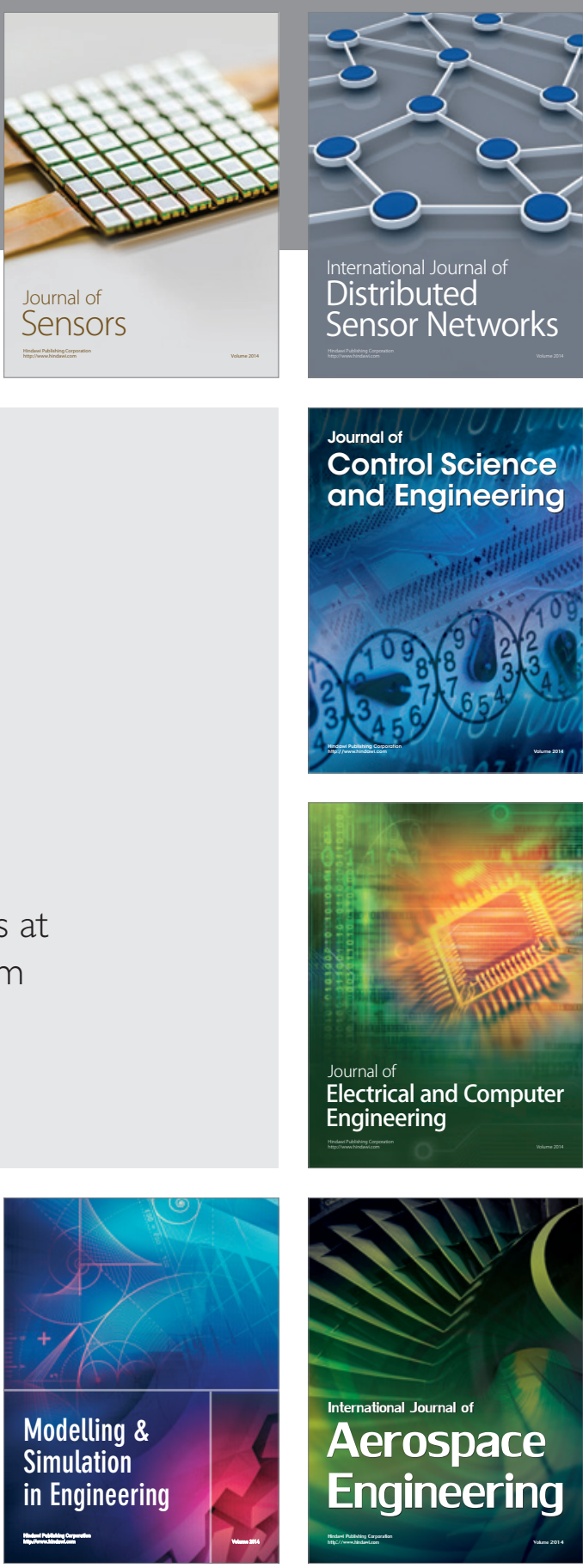

International Journal of

Distributed

Sensor Networks

Journal of

Control Science

and Engineering
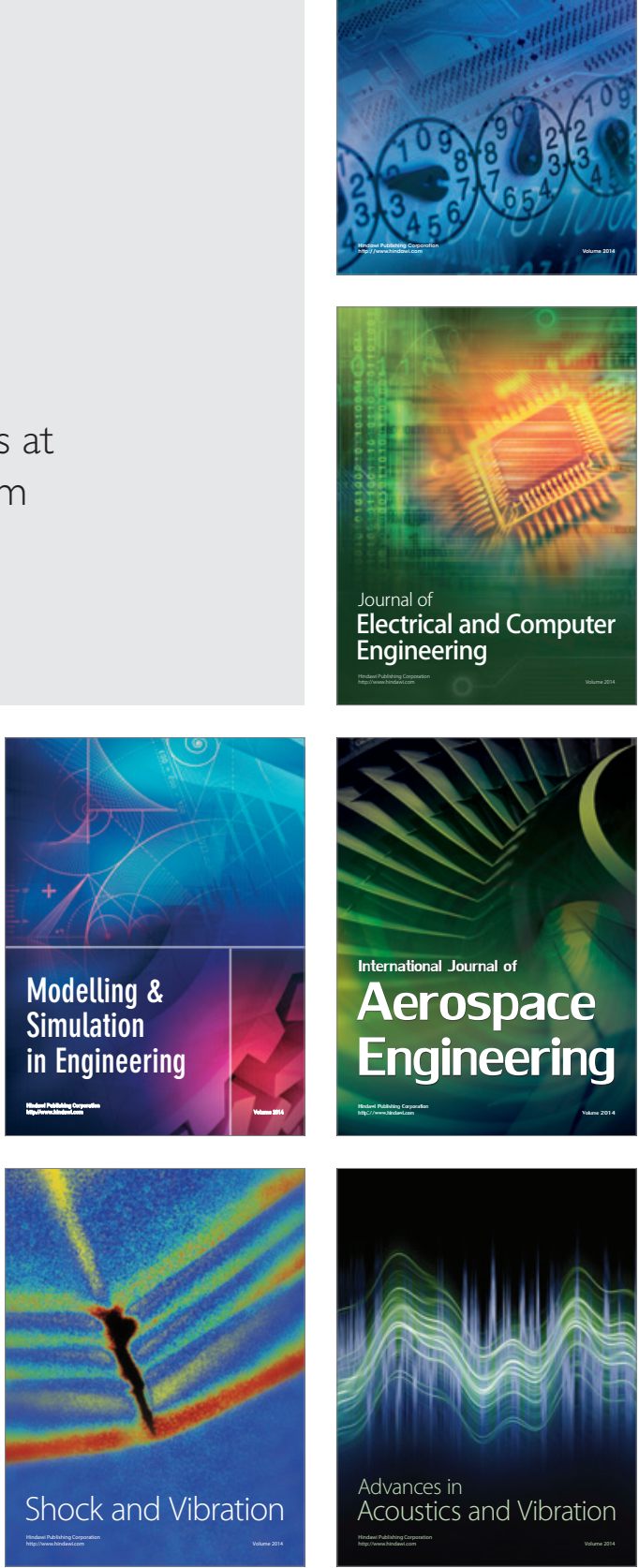